International Journal of Engineering \& Technology, $7(1.2)(2018)$ 61-65
SPC
International Journal of Engineering \& Technology
Website www.sciencepubco.com/index.php/IJET
Research paper

\title{
Optimizing performance of fuzzy decision support system with multiple parameter dependency for cloud provider evaluation
}

\author{
Uma Rani ${ }^{1}$ *, Surjeet Dalal ${ }^{2}$, Jugnesh Kumar 2 \\ ${ }^{1}$ Department of Computer Science \& Engineering, SRM University Sonipat, Haryana, India \\ ${ }^{2}$ Department of Computer Science \& Engineering, St. Andrews Institute of Technology and Management, Haryana \\ *Corresponding author E-mail: profsurjeetdalal@gmail.com
}

\begin{abstract}
In today's world, technological trend offers computing resources as services through the internet in on-demand or pay-as-you-go approach. These services are provided by different cloud service providers. Due to which trust on the any service provider is a choice of any customer. In order to choose a reputed cloud service provider a new method using the concept of fuzzy has proposed in this paper. This method enhanced the customer's satisfaction level of using cloud services by avoiding ambiguities in fuzzy interface system (FIS) through optimization. Proposed fuzzy rule-based decision support system is collaborating with advanced fuzzy system optimized using a swarm intelligent firefly algorithm that facilitates the consumers in selecting right CSP based upon their rating value. It conducts three different reviews of three different components, i.e. customer review, service provider review and public review. Results are carried out on the basis of both simple and the optimized fuzzy, and it is found that the optimized fuzzy surpasses the simple fuzzy logic.
\end{abstract}

Keywords: Decision Support System; Fuzzy Decision Making; Cloud Service Provider.

\section{Introduction}

Cloud computing is a distributed computing model in which the computer facilities and several other resources are provided to the user on the basis of pay as you go criteria [1]. This On-demand model mainly intended to increase the opportunities for the users by using the cloud infrastructure and application hosted in the cloud on the leased basis structure by using these facilities with the help of internet from anywhere. The different kind of information and services are offered by cloud computing in order to expand the visualization of the different IT services [2-3].

A flawless computing can only be achieved in the cloud environment by satisfying the below written requirements

a) Resource Pooling: A service provider providing the cloud services should support the multitenancy of the resource, in order to maximize the efficiency of the infrastructure. For example, it should be able to dynamically assign the resource according to the demand of the consumer.

b) On-demand self-service: It focuses on the available resources that are required by the user. These resources can be the power of the Central Processing Unit or the network requirements. As it is a self service, it is recommended that there is no human interference.

c) A broad network access: As the internet behaves as an interesting medium for user and the system, the availability of the broad network access becomes important for the flawless experience of the user while using the application available on the different cloud platform.

d) Rapid Elasticity: It means the flexibility in order to access the computing resource on the basis of user requirement.
Let us assume that a user wants to reject the resources after completing the work, so there has to be a truthful and flexible commitment so that user can easily rollback from the allocated resources.

e) Measure of Service: A proper system of framework has to be present in order to estimate the usage based on the individual user handling of the resources.

Concluding this part, a cloud service provider is accountable for making the resources accessible on the ondemand basis for the multiple users, accessing that cloud and manages the available resources in a proficient manner in order to achieve the user constraints [10].

\subsection{Trending approaches of inference system}

Trust becomes an important factor while using the distributed system. In such a changing environment, trust is the major concern in order to enhance the interaction between the resource user and the resource providers.

The process of the trust evaluation is clear and uncomplicated for the user base due to the presence of fuzzy and the unique individual values. It guides to the way of a clear picture of the narrative method to give the trust value in a way that can be understood and represented easily. To solve this problem fuzzy logic can be a handy approach [10]. The approach of using the fuzzy logic is a better method in order to describe the human observation. Hence, the approach of fuzzy logic is proposed in this paper in order to estimate the credibility of the service provider that is providing the different resources on the basis of different rating given by the customers. 


\subsection{Benefits}

The Fuzzy inference is a definite procedure from the offered input to the output by utilizing the logic and mapping of fuzzy, that gives the base on the basis of that base the choice has been done, or pattern can be predicted. It is utilized to reproduce or simulate the decision making of human that depends on the fuzzy control rules. To relate the yields of the illative tenets, low-high inference technique is utilized [19] [20].

Lofti A. Zadeh has given the methodology of the fuzzy sets firstly. He presumes that all the issues present in reality could be effortlessly solved with effective and logical methods or by the electronic PCs [15].

It is concluded from several research reports that the service provider state has a high impact on the online sell-off rates, particularly for the high -valued items [1]. The reliance between the customers and service provider"s can get influenced by the reputation construed from agents in their framework or system. By the investigating and designing these systems, this inference has been often hand-waived [1]. In addition, many reviews don't account the probability of extortion and doubt [1].

In his work, Akerlof pointed the primary issue about the data inconsistency between the purchasers and the merchants. The purchasers know their own particular exchanging conduct and the quality of their items they are offering. And the dealers or merchants can assume that what the purchasers know about them from data collected, for example, their status and reputation. The reputation of the trading partners is used with each other to diminish this data asymmetry to encourage the unquestioning of the exchanging connections. [2]

In this paper, an approach based on the fuzzy interface system has developed which explains how the reputation value of the particular service provider can evaluate and how a customer can choose the bestreputed service provider based upon the rating values.

\section{Related work}

Fuzzy logics have been used by different models in order to provide a reliable solution to the online services. Many of the researchers have proposed different ideas, collaborated with the fuzzy system for evaluation of trust on a particular service provider.

For illustration, Falcon et al. [3] proposed a model of socialcognitive approach which evaluates the credibility of a service. It has been used with different components for evaluation and can be altered according to the required situations. Considering the other approach model implemented by Sabater and Sierra [4] defines a fuzzy logic based approach. This approach is used to analyze the relationship of different service users in e-marketplaces or electronic marketplaces. In order to provide secure transmission to the users in the architecture, a mechanism has been used named as the reputation mechanism. This mechanism uses in different e-market systems such as Alibaba and Snapdeal. Accordingly, models have been developed on the basis of trust and reputation, in this paper to provide reliable and secure transmission in e-marketplaces. Alternatively, it does not offer security to the providers and the services consumers.

Md Whaiduzzaman [17] this work analyzed the applications of the MCDA in the area of the selection of services in the Cloud Computing. Along with this, several techniques involved in the same approach were discussed and identified as per the case study dependency. Figures and results are analyzed for the approach defined in the cloud computing. The work of author mainly focused on the brief of cloud computing service selection by working on different case study and scenarios. Along with this a comparative study and analysis of different MCDA approaches was done for the evaluation of the results. Also the study defines the different state of MCDA techniques

Another scenario presented by [8] for the trust quantification was based on a fuzzy comprehensive evaluation theory for the cloud computing, which helps to protect the user"s data through trust quantification of different cloud services. In this paper, the existing trust concept based on dynamic requirements was enhanced. Some cloud service attributes were introduced to study layered service representation for the trust, preference and then applied fuzzy comprehensive evaluation theory to perform the trust quantification.

Carbo [5] in his work developed a trust management system using a fuzzy logic, which prevents unclear, inaccurate and unsure information. Simulation has performed through combining two fuzzy sets named as $\mathrm{S}$ and $\mathrm{R}$ in which $\mathrm{S}$ represents the satisfaction ensures by the merchant and $\mathrm{R}$ represents the reputation ensures by the service provider. Thus, proposed model rotates around the reputation of different merchants and its predictions have been evaluated through buyers of the system. One aspect, i.e. independent view about the behavior of the merchant is vague in this work. Bharadwaj et al. [6] discussed a model in his paper this model is based on the reputation and trust which is basically dependent on the parameters as reciprocity and an experience. Both parameters were evaluated simultaneously, in which reputation is the key parameter to make out similarities and also to discover its neighbors. And the parameter of trust has been evaluated on which the filtering of the neighbors has been done in order to achieve the set of person recommend their services. The proposed models here have been imitated to the movie recommender system, i.e. MovieLens, so as to perform compared with the BETA and EBAY reputation models. As a result proposed model only focuses on the trust and reputation of the entities, but deficient in taking nonbiased opinion about the entities of the system.

The fact of uncertainty in the e-transactions in cloud platform are considered by Nafi et al. [7] in their work. E-commerce architecture has proposed, which introduced a secured trust model based on the encryption and fuzzy logic. This architecture aided in lessening the problems of the existing techniques. The Trustworthiness of the system is calculated through the reputation of the company where a broker is considered as a third party. He is responsible for maintaining the information regarding different companies and provides it to the different clients as well. Consequently, direct experiences of different clients were not considered in the model which lacks the system es performance.

Initially, Lik Mui [23] has surveys existing literatures based upon trust, reputation and reciprocity. Consequently, a computational model was proposed which relies on the sociological and biological understandings of reviewing concepts. This proposed model can be applied in real system scenario for calculations of agents trust and the reputation scores in future.

Simone A. Ludwig [25] developed A trust approach using three sources, based on the fuzzy. A valuation of the proposed system is performed that resulted into the effectiveness and stoutness of the fuzzy approach.

In [18], V. introduced a method which improved the accessibility of the cloud using the concept of fuzzy. This method enhanced the customer"s satisfaction level of using cloud services by avoiding ambiguities in fuzzy interface system (FIS).

The most important aspect of these proposed methods is that they all are based on the fuzzy evaluation and their trust on the past behaviors of the users. But the question of worry is that how a customer can choose a right or reputed CSP by taking a smart decision based on the trust. In this article, a

Decision Support System based on the fuzzy rules has been designed which evaluates rating of five different service providers based on Support, Feasibility, Uptime and value parameters which able the customer to pick right CSP.

\section{Proposed fuzzy rule based expert system}

The proposed system appraises reputation of the Cloud Service Providers based upon three components involved such as:

- Direct experience of customer with different Cloud Service Providers 
- The reputation of the Providers providing the cloud resources

- An independent review of the Cloud Auditor

The result acquired after teaming up three components declared above is termed as the Final rating. In the given model, the rating based on each CSP is getting evaluated on the basis of three parties, specifically, first the Cloud Customer, then the Service Providers, and the Auditors. The customer calculates customer review, the Service Provider calculates service provideres review, and Auditor calculates Auditor reviews.

Based on these reviews, optimization algorithm has applied to obtain a rating of each component individually, such as customer rating, service provider rating and auditor"s rating.

The main idea behind proposing a new method is the evaluation of reputed cloud service providers among various CSPs. A proposed method has been divided into three levels such as:

- Level 1 (collaboration of reviews with fuzzy)

- Level 2 (fuzzy with optimization)

- Level 3 (Fuzzy with final rating)

The central phase of this model relates the information with the preceding behaviors of different customers. The data basis on this was carried as a reputation of those users concerning different parameters such as support, features, uptime and value in the case of customer and service provider"s review, data centers, support, monitoring and APIs in the case of auditor"s reviews. These reviews are used to rate the Cloud Service provider where customers and providers rate each other using statistical ratings. This rating will be considered as a reputable report for an individual user which suggests them whether to proceed with the particular provider or not. Consider an example of Amazon, which uses the scales of 1 , as positive, 0 as neutral and -1 as negative respectively. Different customers use this parameter to evaluate the rating of different service providers on the basis of their experiences.

\subsection{Agenda of proposed technique}

A proposed technique is not a single component; it has aggregated with different techniques to offer a reliable solution to the customers. The Components involved in the proposed techniques are: Cloud computing has evolved into several areas, especially in business ideas where CSP provides services to the users as a value which needs to be paid accordingly. Numerous service providers of cloud are available for last few years, thus the customers need to make a careful decision about choosing a reputed CSP based on several parameters. In addition to this, the fuzzy comprehensive evaluation is about the analysis and the synthesis of fuzzy relationships that involve one or more factors which deals with the vagueness and the subjective judgment of multiple factors according to their importance.

A fuzzy-based trust approach has proposed which is totally based on the rating of the users and making of a wise decision among several service providers. The Cloud Service providers combined with the fuzzy interface system based on the reviews given by the users. As fuzzy is the system which is able to compute uncertain values and provides reliable outputs. Moreover, fuzzy can evaluate the human mind in an efficient way which resulted in efficient results. For the proposed work, fuzzy has combined with the optimization algorithm as:

- A single objective algorithm cannot serve the actual purpose of finding fitness value in multilevel or multi criteria data item forms as clusters.

- To define the notion of optimality in multi criteria optimization.

- To translate the distributed representation into a single structure.

- To translate the experts ${ }^{\text {ee }}$ advice into a symbolic representation of fuzzy.

- To evaluate simple fuzzy rule base method rather than a complex mathematical model in the optimization.
Thus, above reasons clears that optimization algorithm can work well with fuzzy systems. Moreover, from the initial stages, there are a number of customers, service providers and auditor reviews are involved, which will be difficult for the system to attain an individual rating of different components. Defined rules for the different components reviews will be extreme in numbers and becomes complicated to evaluate. But optimization algorithm can resolve the issue by providing a unique solution. From the different optimization algorithms, Firefly algorithm has been chosen because of:

- It is an approach for handling uncertainties in a case where firefly makes a choice in choosing best CSP through fuzzy rules. As control strategies of fireflies are best implemented through fuzzy rules.

- Firefly algorithm will help in choosing the right component based on attractiveness and desirability property or on the basis of rating using fuzzy sets.

These are the components involved in the proposed technique that provides collaborated experience for evaluation of the final rating value.

\section{Results and discussion}

The implementation has been conceded out in the MATLAB software. An online survey conducted in which different users of cloud were approached in order to rate individual Cloud Service Provider based upon four different parameters such as, support, uptime, features and value on a scale of $1-5$, whereas one is considered as Very Poor, two is considered as below average, three is used for the average, and our is considered above average and at last the five rating is for Excellent. Obtained results of the ratings given by the customers get stored in a form of a matrix in the software. After that Simulation is performed and the acquired results are mentioned below.

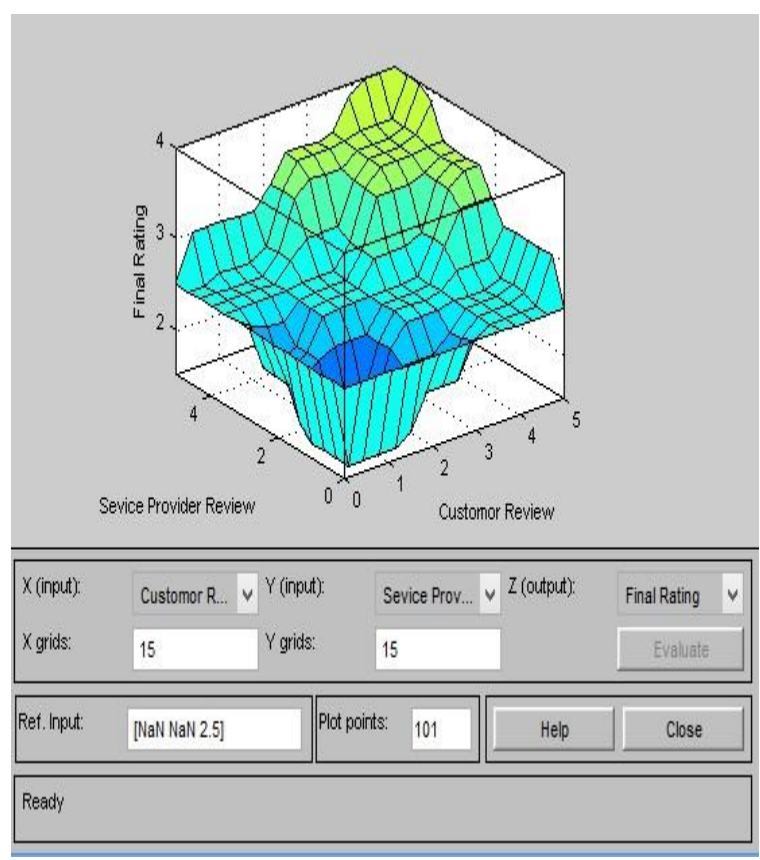

Fig. 1: 3-D Shaded Surface Plots of Reviews Rating.

Above figure represents the surf graph of the proposed model in which different component"s review such as customer reviews and service provider"s review has been shown. These two reviews are input to the system, whereas final rating is the output symbolized as , $Z^{\text {ee }}$. On the basis of inputs, output will be obtained, i.e., aggregated rating of three components. 


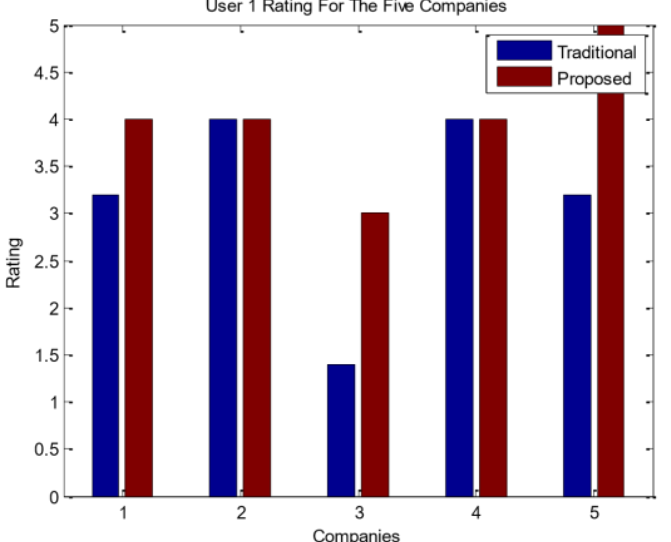

Fig. 2: Rating of User 1 for Five Service Providers.

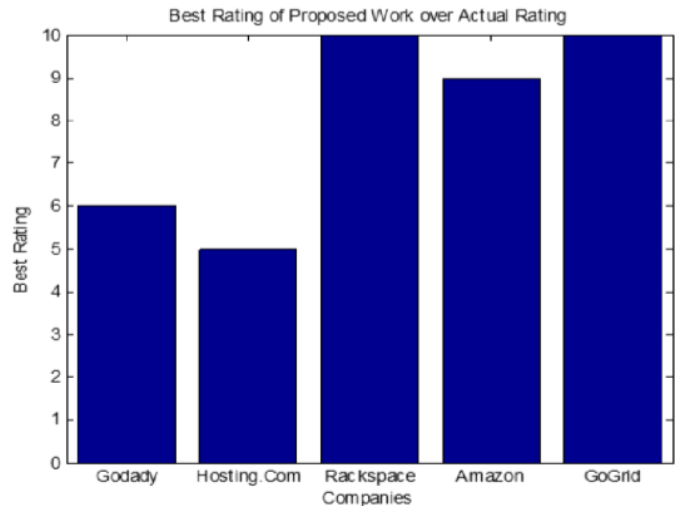

Fig. 3: Best Rating of Proposed Work over Actual Rating.

Rating about individual service provider has taken from the different consumers or users. Each user rates particular service provider accordingly and rating of each customer has shown in terms of bar graphs in figure2. Rating before optimization regarding each company declares as traditional and after optimization affirms as proposed.

Figure 3 below explains the best rating of the proposed work over the actual rating. Thus, the graph contains five service providers with respect to best rating. For each customers rating value, best rating is evaluated.

\section{Conclusion}

The reputation estimation plays an important role in the distributed service oriented environment. Because of the qualities of the service oriented environment and the nature of the trust in the fuzzy, development of a fuzzy-based approach of reputation was created.The developed model calculates the overall rating of the output by taking the three parameters into account as the input parameters i.e customer review, service provider review and the auditor review as the input parameters and. With the joint effort of every segments survey, final rating value has attained. Simulations have been performed, where traditional and proposed rating has compared with each other and it has been concluded that the proposed optimization method is better and feasible for cloud computing. Moreover, on the basis of these ratings of each customer, a decision can be taken in an effective manner regarding a best CSP. In future multiple QoS metrics can be aimed for ensuring the trust of service provider to the customer.

\section{References}

[1] D. E. Houser and John wooders, "Reputation in Internet Auctions Theory and Evidence from eBay," Journal of Economics \& Management Strategy. https://doi.org/10.1111/j.15309134.2006.00103.x.
[2] G. Akerlof, "The Market for „Lemons": Qualitative Uncertainty and the Market Mechanism," Quarterly Journal of Economics, vol. 84, pp. 488-500, 1970. https://doi.org/10.2307/1879431.

[3] R. Falcone, Giovanni Pezzulo and Cristiano Castelfranchi, "A fuzzy approach to a belief-based trust computation," Trust, reputation, and security: theories and practice, pp. 55-60, 2003.

[4] J. Sabater and C. Sierra, "Reputation and social network analysis in multi-agent systems," pp. 475-482, 2002. https://doi.org/10.1145/544741.544854.

[5] Carbo, J., Molina, J.M., Davila, J.: Trust management through fuzzy reputation. International Journal of Coop. Inf. Syst. Vol. 12, no. $01, \quad$ Pp. 2003. https://doi.org/10.1142/S0218843003000681.

[6] Bharadwaj, K.K., Al-Shamri, M.Y.H.: Fuzzy computational models for trust and reputation systems. Electron. Commer. Res. Appl., vol. 8, no. $1, \quad$ pp. $37-47, \quad 2009$ https://doi.org/10.1016/j.elerap.2008.08.001.

[7] Nafi, K.W., Kar, T.S., Hossain, M., Hashem, M.M.A.: A new trusted and secured E-commerce architecture for cloud computing. In: Proceedings of International Conference on Informatics, Electronics \& Vision (ICIEV). IEEE, pp. 1-6, 2013.

[8] Robert H. Bonczek, Clyde W. Holsapple and Andrew B. Whinston, "The evolving roles of models in decision support systems", Vol 11, No. 2, Pp. 337-356, April 1980. https://doi.org/10.1111/j.15405915.1980.tb01143.x.

[9] Ramesh Sharda, Steve H. Barr and James C. MCDonnell, "Decision Support System Effectiveness: A Review and an Empirical Test", Management science, vol. 34, No. 2, Pp. 139-159, February 1988. https://doi.org/10.1287/mnsc.34.2.139.

[10] Mohammed Alhamad, Tharam Dillon, and Elizabeth Chang, "A Trust-Evaluation Metric for Cloud applications”, IJMLC, Vol. 1, No. 4, Pp. 416, October 2011. https://doi.org/10.7763/IJMLC.2011.V1.62

[11] M. Balazinski, E. Czogala and S. Gravelle, "Automatic Tool Selection using a Fuzzy Decision Support System”, IEEE, Pp. 615-620, 1995. https://doi.org/10.1109/FUZZY.1995.409748.

[12] Javier Puente, Raul Pino, Paolo Priore and David de la Fuente, "A Decision Support System for applying failure mode and effects analysis", International Journal of Quality and Reliability management, Vol. 19, No. 2, Pp. 137-150, 2002. https://doi.org/10.1108/02656710210413480.

[13] Ludmil Mikhailov and M.G. Singh, "Fuzzy Analytic Network Process and its Application to the Development of Decision Support Systems", Vol. 33, No. 1, Pp. 33-41, February 2003. https://doi.org/10.1109/TSMCC.2003.809354

[14] Nader Naderpajouh, A. Afshar and S.A. Mirmohammadsadeghi, "Fuzzy Decision Support System for Application of Value

Engineering in Construction Industry", International Journal of Civil Engineering, Vol. 4, No. 4,Pp. 261-273, December 2006.

[15] M. Baran Pouyan R. Yousefi, S. Ostadabbas and M. Nourani, "A Hybrid Fuzzy-Firefly Approach for Rule-Based Classification”, Proceedings of the Twenty-Seventh International Florida Artificial Intelligence Research Society Conference, Pp. 357-362.

[16] Xiaohui Li, Jingsha He, Bin Zhao, Jing Fang, Yixuan Zhang and Hongxing Liang, "A Method for Trust Quantification in Cloud Computing Environments", IJDSN, February 2016. https://doi.org/10.1155/2016/5052614.

[17] Md Whaiduzzaman Abdullah Gani, Nor Badrul Anuar, Muhammad Shiraz, Mohammad Nazmul Haque, and Israat Tanzeena Haque, "Cloud Service Selection Using Multicriteria Decision Analysis", HINDAWI, Vol. 2014, Pages 10, 2014 https://doi.org/10.1155/2014/459375.

[18] V. Prasath, Nithya Bharathan, Neetha N.P, N. Lakshmi and M.Nathiya, "Fuzzy Logic In Cloud Computing", IJERT, Vol. 2, No. 3, March 2013. E-ISSN: 2278-0181.

[19] Qi Zhang, Lu Cheng and Raouf Boutaba, "Cloud computing: stateof-the-art and research challenges", Journal of Internet Services and Applications, vol. 1, no. 1, Pp. 7-18, May 2010. https://doi.org/10.1007/s13174-010-0007-6.

[20] Ilango Sriram and Ali Khajeh-Hosseini, "Research Agenda in Cloud Technologies", 2007.

[21] Srinivas Sethi, Anupama Sahu and Suvendu Kumar Jena, "Efficient load Balancing in Cloud Computing using Fuzzy Logic", IOSRJEN, Vol. 2, No. 7, Pp. 65-71, July, 2012. ISSN: 2250-3021.

[22] Rachna Satsang, Dr. Pankaj Dashore and Dr.Nishith Dubey, "Risk Management in Cloud Computing Through Fuzzy Logic", Vol. 1, No. 4, Pp. 1-4, December 2012. ISSN 2319-4847.

[23] Lik Mui and Ari Halberstadt, "A Computational Model of Trust and Reputation", IEEE, 2002. 
[24] Sini Ruohomaa and Lea Kutvonen, "Trust Management Survey", SPRINGER, vol. 3477, pp. 77-92, 2005.

[25] Ludwig, S.A. Venkat Pulimi and Andriy Hnativ, "Fuzzy approach for the evaluation of trust and reputation of services", IEEE international conference on Fuzzy systems, pp. 115-120, 2009. https://doi.org/10.1109/FUZZY.2009.5277061.

[26] A. Hegyi, B. De Schutter, S. Hoogendoorn, R. Babu`ska, H. van Zuylen, and H. Schuurman, “A Fuzzy Decision Support System for Traffic Control Centers”, Pp. 358-363, August 2001. 